\title{
Modulation of the Expression of Long-Term Cardiac Memory by Short-Term Cardiac Memory in Patients With Wolff-Parkinson-White Syndrome After Catheter Ablation
}

\author{
Kun-Tai Lee, MD*,**; Chih-Sheng Chu, MD**; YE-Hsu Lu, MD**; \\ Tsung-Hsien Lin, MD*,**; Hsueh-Wei Yen, MD*,**; Wen-Chol Voon, MD*,**; \\ Sheng-Hsiung Sheu, MD*,**; Wen-Ter Lai, MD*,**
}

\begin{abstract}
Background The interaction between long- and short-term cardiac memory (CM) is unknown.
Methods and Results The T-wave areas and QTc intervals in each ECG lead were analyzed in 11 patients with manifest Wolff-Parkinson-White syndrome with posterior or septal accessory pathway (4 females; mean age: $47 \pm 12$ years) in the following ECGs: (1) immediately after catheter ablation (post-ablation ECG); (2) immediately after $20 \mathrm{~min}$ of right ventricular outlet pacing (post-pacing ECG); and (3) 1 week after ablation (recovery ECG). Compared with the post-ablation ECGs, the T-wave areas of the recovery ECGs in leads II and aVF changed dramatically from negative to positive while that in lead III became less negative $(\mathrm{p}<0.01)$, and those in leads I, a $V_{L}$, and $V_{2-4}$ became less positive $(\mathrm{p}<0.05)$. Compared with the post-ablation ECGs, the T-wave areas of the post-pacing ECGs in leads III and $\mathrm{aVF}$ became less negative $(\mathrm{p}<0.01)$, and those in leads $\mathrm{I}_{\mathrm{a}} \mathrm{a} \mathrm{L}$, and $\mathrm{V}_{2-4}$ became less positive $(\mathrm{p}<0.05)$. The QTc interval in the post-ablation ECG was significantly longer than in either the post-pacing or recovery ECGs $(\mathrm{p}<0.05)$.

Conclusions Mechanisms involved in the expression of long-term CM could be affected by short-term CM.
\end{abstract} (Circ J 2007; 71: 331-337)

Key Words: Cardiac memory; ECG; Wolff-Parkinson-White syndrome

A fter catheter ablation in patients with manifest Wolff-Parkinson-White (WPW) syndrome, significant changes of $\mathrm{T}$-wave polarity in the 12-lead ECG are often demonstrated ${ }^{1-3}$ but they gradually normalize over weeks. Kalbfleisch et al attributed these T-wave changes to cardiac memory (CM) resulting from ventricular preexcitation before catheter ablation? $\mathrm{CM}$ is characterized by changes in the T-wave vector on ECG that follow resumption of sinus rhythm after a period of altered ventricular activation sequence.,5 The direction of the change in the T-wave vector is in that of the vector of the inciting and abnormally activated QRS complex. CM demonstrated in patients with WPW syndrome after catheter ablation has been referred to as "long-term" CM because of the long period of ventricular preexcitation that altered the ventricular activation sequences. Alternatively, CM can be induced by a short period of alternating ventricular depolarization by ventricular pacing and is referred to as "short-term" CM6 The mechanisms of CM are complex and have been widely explored in animal studies?-15 In humans, although expression of long- and short-term CM has been demonstrated,

(Received July 26, 2006; revised manuscript received November 22, 2006; accepted December 7, 2006)

*Department of Internal Medicine, College of Medicine, Kaohsiung Medical University, **Division of Cardiology, Department of Internal Medicine, Kaohsiung Medical University Hospital, Kaohsiung, Taiwan Mailing address: Wen-Ter Lai, MD, Division of Cardiology, Department of Internal Medicine, Kaohsiung Medical University Hospital, 100 Tzyou 1ST RD. Kaohsiung, 80708, Taiwan. E-mail: kuntai.lee @msa.hinet.net the mechanisms of $\mathrm{CM}$ are still unclear., ${ }^{8}$ In addition, the interaction between short- and long-term CM has not been reported before. The aim of this study was to analyze whether the induction of short-term CM affects the expression of existing long-term CM in humans.

\section{Methods}

The study protocol was approved by the Institutional Review Board at Kaohsiung Medical University. Informed consent was given by all patients.

\section{Patients}

In order to obtain inverted $\mathrm{T}$-waves that represented long-term CM in leads II, III and $\mathrm{aVF}_{\mathrm{F}}$ after catheter ablation, 11 patients with manifest WPW syndrome (4 females, 7 males; mean age: $47 \pm 12$ years) with a posterior or septal accessory pathway (AP) who had undergone catheter ablation were enrolled. Before the electrophysiological studies and catheter ablation, all patients underwent routine physical examination and echocardiography, and no significant organic heart disease was demonstrated.

\section{Electrophysiological Study and Radiofrequency Catheter Ablation}

Antiarrhythmic drugs were stopped at least 5 half-lives before the commencement of the electrophysiological study and catheter ablation, which were performed in the unsedated and postabsorptive state. For recording and stimulation, 3 quadripolar and 1 decapolar electrode catheters 
A

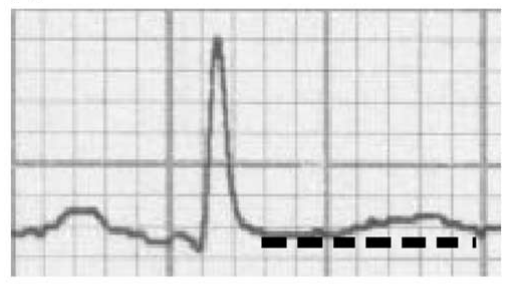

B

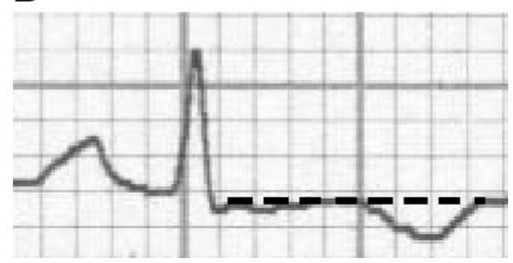

Fig 1. Measurement of the T-wave area, defined as the area between the $\mathrm{J}$-T line (dashed line) and the $\mathrm{T}$-wave. The $\mathrm{T}$-wave area above the $\mathrm{J}$-T line was defined as positive (A), and below the J-T line as negative (B).

were introduced into the right femoral vein and advanced to the high right atrium, right atrioventricular junction, right ventricular apex (RVA), and the coronary sinus, respectively. The 12-lead surface ECG and bipolar intracardiac ECGs were recorded and stored digitally (Bard LabSystem EP Laboratory, Lowsell, MA, USA) for retrospective study. After the electrophysiological characteristics of the AP were determined, radiofrequency catheter ablation was performed using a 7-Fr steerable, quadripolar electrode catheter with a 4-mm tip electrode (Stinger, C.R. Bard, Inc), which was introduced percutaneously via the right femoral vein or artery and advanced to the tricuspid or mitral ring, respectively. The location of the fusion of atrial and ventricular electrical activity was mapped as the ablation site. A temperaturecontrolled radiofrequency ablation unit (EPT-1000TC; EP Technologies, Mountain View, CA, USA) was used with the ablation temperature set at $60^{\circ} \mathrm{C}$ and duration at $60 \mathrm{~s}$. The endpoint of ablation was defined as the absence of anterograde and retrograde AP conduction, as confirmed by the electrophysiological study.

\section{Induced Short-Term CM}

Before catheter ablation, the QRS polarity in each surface ECG lead was determined as positive, negative or positive/negative according to the morphology of the QRS. Immediately after catheter ablation, the 12-lead ECG was recorded as the post-ablation ECG. The QRS polarity, characteristics of the T-waves and QT interval in each lead were analyzed. Results of a previous study had shown that short-term CM could be induced by 20-min of pacing from the right ventricle $(\mathrm{RV}){ }^{13}$ In order to create a positive polarity of the QRS complex in surface leads II, III and aVF during RV pacing to counteract the negative T-wave polarity induced by long-term CM, the RVA catheter was repositioned at the right ventricular outlet tract (RVOT) area. Sustained pacing with rectangular pulses of 2-ms duration at 2-fold the diastolic threshold from the RVOT was intro-

Table 1 Location of Accessory Pathway and QRS Complex Polarity in the Limb Leads Before and After Catheter Ablation

\begin{tabular}{|c|c|c|c|c|c|c|c|c|c|c|c|c|c|}
\hline \multirow{2}{*}{ Case no. } & \multirow{2}{*}{$A P$} & \multicolumn{2}{|c|}{$I$} & \multicolumn{2}{|c|}{ II } & \multicolumn{2}{|c|}{ III } & \multicolumn{2}{|c|}{$a V R$} & \multicolumn{2}{|c|}{$a V_{L}$} & \multicolumn{2}{|c|}{$\bar{a} a V_{F}$} \\
\hline & & $B-A B$ & $P-A B$ & $B-A B$ & $P-A B$ & $B-A B$ & $P-A B$ & $B-A B$ & $P-A B$ & $B-A B$ & $P-A B$ & $B-A B$ & $P-A B$ \\
\hline 1 & $R P$ & $P$ & $P$ & $N / P$ & $P$ & $N$ & $P$ & $N$ & $N$ & $P$ & $P / N$ & $N$ & $P$ \\
\hline 2 & $R P$ & $P$ & $P / N$ & $N / P$ & $P$ & $N$ & $P$ & $N$ & $N$ & $P$ & $P / N$ & $N$ & $P$ \\
\hline 3 & $R P S$ & $P$ & $P$ & $P / N$ & $P$ & $N$ & $P$ & $N$ & $N$ & $P$ & $P / N$ & $P / N$ & $P$ \\
\hline 4 & $R P S$ & $P$ & $P$ & $N$ & $P$ & $N$ & $P$ & $N$ & $N$ & $P$ & $P / N$ & $P / N$ & $P$ \\
\hline 5 & $R L$ & $P$ & $P$ & $P$ & $P$ & $N / P$ & $P$ & $N$ & $N$ & $P$ & $P$ & $P$ & $P$ \\
\hline 6 & $L P$ & $P$ & $P$ & $P$ & $P$ & $N$ & $P$ & $N$ & $N$ & $P$ & $N / P$ & $P / N$ & $P$ \\
\hline 7 & $R P$ & $P$ & $P$ & $P$ & $P$ & $N$ & $P$ & $N$ & $N$ & $P$ & $P / N$ & $N$ & $P$ \\
\hline 8 & $R P$ & $P$ & $P$ & $P$ & $P / N$ & $N$ & $P$ & $N$ & $N$ & $P$ & $P$ & $N$ & $P$ \\
\hline 9 & $R P$ & $P$ & $P / N$ & $N / P$ & $P$ & $N$ & $P$ & $N$ & $N$ & $P$ & $N$ & $P$ & $P$ \\
\hline 10 & $R P S$ & $P$ & $P$ & $N$ & $P$ & $N$ & $P / N$ & $N$ & $N$ & $P$ & $P$ & $N$ & $P / N$ \\
\hline 11 & $L P$ & $P$ & $P / N$ & $P / N$ & $P$ & $N$ & $P$ & $N$ & $N$ & $P$ & $P / N$ & $N / P$ & $P$ \\
\hline
\end{tabular}

$A P$, location of accessory pathway; $B-A B$, before ablation; $P-A B$, post-ablation; $R P$, right posterior; $P$, positive; $N$, negative; RPS, right posterior septum; $R L$, right lateral; $L P$, left posterior.

Table 2 QRS Complex Polarity in the Precordial ECG Before and After Catheter Ablation

\begin{tabular}{|c|c|c|c|c|c|c|c|c|c|c|c|c|}
\hline \multirow{2}{*}{ Case no. } & \multicolumn{2}{|c|}{$V_{l}$} & \multicolumn{2}{|c|}{$V_{2}$} & \multicolumn{2}{|c|}{$V_{3}$} & \multicolumn{2}{|c|}{$V_{4}$} & \multicolumn{2}{|c|}{$V_{5}$} & \multicolumn{2}{|c|}{$V_{6}$} \\
\hline & $B-A B$ & $P-A B$ & $B-A B$ & $P-A B$ & $B-A B$ & $P-A B$ & $B-A B$ & $P-A B$ & $B-A B$ & $P-A B$ & $B-A B$ & $P-A B$ \\
\hline 1 & $N$ & $P / N$ & $P$ & $P / N$ & $P$ & $P / N$ & $P$ & $P / N$ & $P$ & $P / N$ & $P$ & $P$ \\
\hline 2 & $N$ & $P / N$ & $P$ & $P / N$ & $P$ & $P / N$ & $P$ & $P / N$ & $P$ & $P / N$ & $P$ & $P$ \\
\hline 3 & $N$ & $P / N$ & $N$ & $P / N$ & $N$ & $P / N$ & $P / N$ & $P / N$ & $P / N$ & $P / N$ & $P$ & $P$ \\
\hline 4 & $N$ & $P / N$ & $N$ & $P / N$ & $N$ & $P / N$ & $P / N$ & $P / N$ & $P / N$ & $P / N$ & $P$ & $P$ \\
\hline 5 & $P / N$ & $P / N$ & $P$ & $P / N$ & $P$ & $P / N$ & $P$ & $P$ & $P$ & $P$ & $P$ & $P$ \\
\hline 6 & $P / N$ & $P / N$ & $P$ & $P / N$ & $P$ & $P / N$ & $P$ & $P$ & $P$ & $P$ & $P$ & $P$ \\
\hline 7 & $N$ & $P / N$ & $P / N$ & $P / N$ & $P / N$ & $P / N$ & $P$ & $P / N$ & $P$ & $P$ & $P$ & $P$ \\
\hline 8 & $N$ & $P / N$ & $P$ & $P / N$ & $P$ & $P / N$ & $P$ & $P / N$ & $P$ & $P$ & $P$ & $P$ \\
\hline 9 & $N$ & $P / N$ & $P / N$ & $P / N$ & $P$ & $P / N$ & $P$ & $P / N$ & $P$ & $P / N$ & $P$ & $P$ \\
\hline 10 & $N$ & $P / N$ & $P$ & $P / N$ & $P$ & $P / N$ & $P$ & $P / N$ & $P$ & $P / N$ & $P$ & $P / N$ \\
\hline 11 & $P$ & $P$ & $P$ & $P$ & $P$ & $P$ & $P / N$ & $P / N$ & $P / N$ & $P / N$ & $P / N$ & $P / N$ \\
\hline
\end{tabular}

Abbreviations as in Table 1. 
Table 3 T-Wave Areas of the Post-Ablation and Recovery ECGs, and LCM in the Limb Leads

\begin{tabular}{|c|c|c|c|c|c|c|c|c|c|c|c|c|c|c|c|c|c|c|}
\hline \multirow{2}{*}{ Case no. } & \multicolumn{3}{|c|}{$I\left(\mathrm{~mm}^{2}\right)$} & \multicolumn{3}{|c|}{ II $\left(\mathrm{mm}^{2}\right)$} & \multicolumn{3}{|c|}{ III $\left(\mathrm{mm}^{2}\right)$} & \multicolumn{3}{|c|}{$\overline{a V R}\left(\mathrm{~mm}^{2}\right)$} & \multicolumn{3}{|c|}{$a V_{L}\left(m^{2}\right)$} & \multicolumn{3}{|c|}{$a V_{F}\left(m m^{2}\right)$} \\
\hline & $P-A B$ & $R C$ & $\overline{C M}$ & $A B$ & $R C$ & $L C M$ & $A B$ & $R C$ & $L C M$ & $A B$ & $R C$ & $L C M$ & $-A B$ & $R C$ & $L C$ & $P-A B$ & $R C$ & $\overline{L C M}$ \\
\hline 1 & 31 & & 7.9 & 7 & 7. & 7 & 16 & 1.84 & 30 & .97 & 50 & 47 & 63 & 3.3 & 1.33 & 5.51 & 4.89 & -10.40 \\
\hline 2 & & & 2. & 35 & & -7.24 & & 1.93 & & -7.57 & -7.51 & -0.06 & & & 6.75 & -4.63 & 4.90 & -9.53 \\
\hline 3 & & & 26.21 & -10.8 & -0.57 & -10.23 & -39.78 & -4.25 & & -11.18 & & & & & 2.85 & 52 & & 02 \\
\hline . & 16.86 & 11.1 & 571 & 7.01 & 11.03 & -4.02 & & -2.24 & -6.06 & -12.78 & -10.16 & -2 . & & & 7.10 & 48 & 70 & -6.18 \\
\hline 5 & & & & 4.48 & & -1.96 & -2.98 & 2.75 & -5.73 & -5.56 & -6.73 & 1.17 & 20 & & 3.66 & 2.23 & 4.47 & -2.24 \\
\hline 6 & 56 & 0. & 2.66 & -3.04 & 1.56 & -4.60 & -11.40 & -4.56 & -6.84 & -3.38 & -3.71 & 0.33 & 9.91 & 5. & 4.56 & -7.65 & -2.41 & -5.24 \\
\hline 7 & 7.56 & 2.50 & 5.06 & 5.72 & 4.54 & 1.18 & -4.85 & 1.25 & -6.10 & -6.62 & -2.65 & -3.97 & 5.66 & 0. & 5.11 & 0.40 & 3.39 & -2.99 \\
\hline 8 & 11.80 & 5.7 & 6.08 & -2.35 & 6.65 & -9.0 & -13.47 & 0.0 & -13.47 & -5.42 & -6.13 & 0.71 & 12.83 & 3. & 9.79 & -7.58 & 3.73 & -11.31 \\
\hline 9 & & & & & 6.3 & -9.58 & 3 & & -10.00 & -1.68 & -5.36 & 3.6 & .79 & & 94 & -5.34 & 13 & -9.47 \\
\hline 10 & & & & -6.33 & 3.69 & -10.02 & -12.55 & -3.75 & -8.80 & 0.0 & -4.47 & 4. & 8.99 & 6. & 92 & -9.89 & -1.18 & -8.71 \\
\hline 1 & 9.19 & & 3. & -1.60 & 3.9 & -5.58 & -9.63 & -2.86 & -6.77 & -5.36 & -4.90 & -0.46 & 10.16 & 5. & 5.09 & -5.29 & 0.0 & -5.29 \\
\hline Mean & 12.09 & 6.1 & 5.92 & -0.77 & 5.34 & -6.11 & -12.05 & -0.67 & -11.39 & -6.14 & -5.50 & -0.64 & 12.33 & 3.67 & 8.65 & -6.21 & 2.19 & -8.40 \\
\hline SEM & 2.24 & 0.74 & 2.13 & 1.60 & 0.96 & 1.09 & 2.94 & 0.87 & 2.58 & 1.14 & 0.75 & 1.17 & 2.64 & 0.57 & 2.53 & 2.04 & 0.92 & 1.56 \\
\hline$p$ value & 0.003 & & & 0.004 & & & 0.003 & & & 0.86 & & & 0.003 & & & 0.003 & & \\
\hline
\end{tabular}

$R C$, recovery; $L C M$, long-term cardiac memory. Other abbreviations as in Table 1.

$L C M=(P-A B)-(R C) . P$ value of comparison between $P-A B$ and $R C$.

Table 4 T-Wave Areas of the Post-Ablation and Recovery ECGs, and LCM in the Precordial Leads

\begin{tabular}{|c|c|c|c|c|c|c|c|c|c|c|c|c|c|c|c|c|c|c|}
\hline \multirow{2}{*}{ Case no. } & \multicolumn{3}{|c|}{$V_{l}\left(m m^{2}\right)$} & \multicolumn{3}{|c|}{$V_{2}\left(\mathrm{~mm}^{2}\right)$} & \multicolumn{3}{|c|}{$V_{3}\left(\mathrm{~mm}^{2}\right)$} & \multicolumn{3}{|c|}{$V_{4}\left(\mathrm{~mm}^{2}\right)$} & \multicolumn{3}{|c|}{$V_{5}\left(\mathrm{~mm}^{2}\right)$} & \multicolumn{3}{|c|}{$V_{6}\left(\mathrm{~mm}^{2}\right)$} \\
\hline & $P-A B$ & $R C$ & $L C M$ & $P-A B$ & $R C$ & $L C M$ & $P-A B$ & $R C$ & $L C M$ & $P-A B$ & $R C$ & $L C M$ & $P-A B$ & $R C$ & $L C M$ & $P-A B$ & $R C$ & $L C M$ \\
\hline 1 & -4.57 & -2.59 & -1.98 & 28.61 & 14.98 & 13.63 & 37.17 & 19.98 & 17.18 & 36.63 & 22.59 & 14.04 & 24.70 & 18.73 & 5.97 & 17.91 & 15.14 & 2.77 \\
\hline 2 & -5.04 & -2.50 & -2.54 & 14.15 & 15.03 & -0.88 & 22.11 & 19.90 & 2.08 & 21.11 & 22.64 & -1.53 & 18.13 & 18.70 & -0.57 & 14.50 & 15.00 & -0.50 \\
\hline 3 & -2.98 & 1.79 & -4.77 & 47.55 & 11.88 & 35.67 & 42.67 & 10.19 & 32.48 & 23.17 & 6.21 & 16.96 & 27.58 & 6.72 & 20.86 & 17.73 & 5.55 & 12.18 \\
\hline 4 & -5.42 & -3.19 & -2.23 & 36.89 & 8.60 & 28.29 & 40.58 & 10.03 & 30.58 & 33.27 & 10.01 & 23.26 & 20.84 & 9.33 & 11.51 & 13.05 & 10.00 & 3.05 \\
\hline 5 & -3.72 & 1.58 & -5.30 & 19.55 & 6.80 & 12.75 & 10.82 & 7.39 & 3.43 & 9.05 & 4.91 & 4.14 & 7.00 & 4.42 & 2.58 & 6.87 & 3.46 & 3.41 \\
\hline 6 & 16.48 & -0.58 & 17.06 & 46.91 & 7.50 & 39.41 & 31.83 & 4.79 & 27.04 & 17.63 & 1.58 & 16.05 & 7.29 & 2.05 & 5.24 & 2.65 & 1.52 & 1.13 \\
\hline 7 & -8.48 & 3.12 & -11.60 & -6.75 & 5.50 & -12.25 & 0.9 & 6.98 & -6.08 & 9.68 & 6.64 & 3.04 & 13.29 & 6.05 & 7.24 & 11.31 & 4.32 & 6.99 \\
\hline 8 & -1.10 & -1.93 & 0.83 & 31.41 & 17.57 & 13.84 & 30.94 & 15.01 & 15.94 & 22.56 & 10.88 & 11.68 & 12.27 & 9.95 & 2.32 & 6.10 & 7.88 & -1.78 \\
\hline 9 & 8.92 & 2.44 & 6.48 & 20.84 & 5.18 & 15.66 & 11.73 & 4.15 & 7.58 & 6.80 & 9.37 & -2.57 & 4.49 & 8.58 & -4.09 & 2.47 & 7.33 & -4.86 \\
\hline 10 & 3.86 & 3.02 & 0.84 & 18.73 & 11.09 & 7.64 & 15.47 & 14.79 & 0.68 & 11.91 & 10.23 & 1.68 & 3.54 & 8.39 & -4.85 & -2.73 & 5.35 & -8.08 \\
\hline 11 & -11.29 & -11.34 & 0.05 & -8.37 & -13.91 & 5.54 & -6.29 & -5.1 & -1.19 & 6.47 & 3.96 & 2.51 & 5.29 & 5.28 & 0.01 & 4.53 & 5.02 & -0.49 \\
\hline Mean & -1.21 & -0.93 & -0.29 & 22.68 & 8.20 & 14.48 & 21.63 & 9.84 & 11.79 & 18.03 & 9.91 & 8.11 & 13.13 & 8.93 & 4.20 & 8.58 & 7.32 & 1.26 \\
\hline SEM & 2.43 & 1.27 & 2.20 & 5.60 & 2.54 & 4.62 & 4.95 & 2.24 & 4.10 & 3.14 & 2.09 & 2.59 & 2.57 & 1.61 & 2.22 & 2.04 & 1.34 & 1.66 \\
\hline$p$ value & 0.53 & & & 0.01 & & & 0.02 & & & 0.01 & & & 0.07 & & & 0.47 & & \\
\hline
\end{tabular}

Abbreviations as in Tables 1,3.

duced. The pacing cycle length was set at $400 \mathrm{~ms}$ and the absence of a sinus capture beat was confirmed. Immediately after RVOT pacing for $20 \mathrm{~min}$, the 12-lead ECG was recorded and referred to as the post-pacing ECG. At 1 week after the ablation procedure, another 12-lead ECG was obtained and was referred to as the recovery ECG.

\section{Measurement of T-Wave Area and QT Interval}

For quantification of CM, both the T-wave area and maximal corrected QT interval (QTc) were measured on the (1) post-ablation, (2) post-pacing and (3) recovery ECGs. The T-wave area was defined as the area surrounded by the T-wave and the line between the $\mathrm{J}$ point and the end of the T-wave (J-T line). The value of the T-wave area above the $\mathrm{J}$-T line was defined as positive and below the J-T line as negative (Fig 1). Representative 12-lead ECG recordings from among the post-ablation, post-pacing and recovery ECGs were saved to a personal computer. The T-wave area, QT intervals and RR intervals were measured by software (Image Tool version 2.0; UTHSCSA, San Antonio, TX, USA) using a caliber on the computer. The mean T-wave area, QT interval and RR interval were obtained by measuring the T-wave areas, QT intervals and RR intervals of 3 consecutive sinus beats in each lead.

\section{Definitions}

The recovery ECG was defined as the "baseline" ECG and the expression of long-term CM was defined as the difference in the mean T-wave area between the post-ablation and recovery ECGs in each lead; that is, the mean T-wave area of the post-ablation ECG minus that of the recovery ECG. A positive value indicated that the T-wave area became less positive or more negative in the recovery ECG compared with the post-ablation ECG. The effects of shortterm $\mathrm{CM}$ on the expression of long-term $\mathrm{CM}$ were defined as the difference in the mean T-wave area between the post-pacing and post-ablation ECGs in each lead; that is, the mean T-wave area of the post-pacing ECG minus that of the post-ablation ECG. A positive value indicated that the T-wave area became less negative or more positive after short-term pacing. The maximal QTc was calculated by the Bazett method.

\section{Statistical Analysis}

All data were analyzed using SPSS version 11.0 (SPSS Inc, Chicago, IL, USA). Results are presented as mean \pm SEM. Comparisons of mean T-wave area and maximal QTc in each lead were made using the Wilcoxon test. A p-value of less than 0.05 was considered statistically significant. 
A

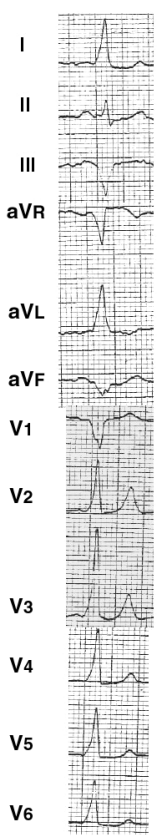

B

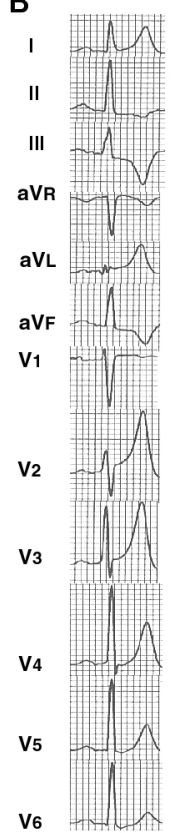

C

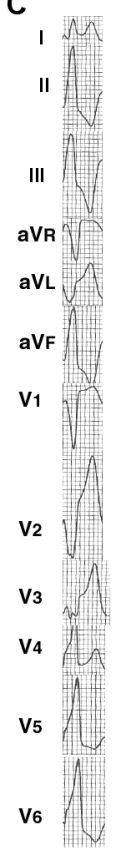

D

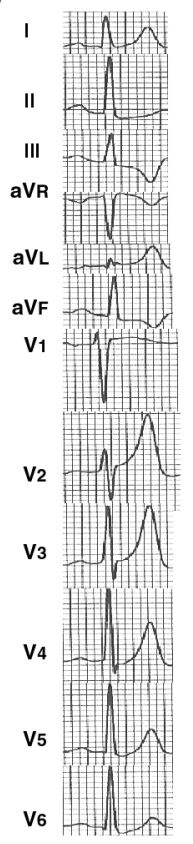

E

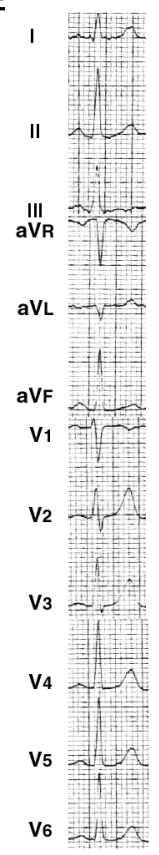

Fig 2. Effect of short-term cardiac memory $(\mathrm{CM})$ on long-term $\mathrm{CM}$ in patient 8 . Before catheter ablation, the patient had an atrioventricular accessory pathway (AP) on the right posterior wall (A). After AP conduction is completely abolished, long-term CM is expressed and the T-wave areas become negative in leads II, III, and $\mathrm{aVF}(\mathrm{B})$. The polarity of the QRS complexes is positive in leads II, III, and $\mathrm{aVF}$ during right ventricular outlet tract (RVOT) pacing (C). After $20 \mathrm{~min}$ of RVOT pacing, the T-wave areas become less negative in leads II, III and $\mathrm{aVF}_{\mathrm{F}}$ (D). The Twave areas become positive in leads II, III and $\mathrm{aVF}$ at 1 week after the ablation procedure (E).

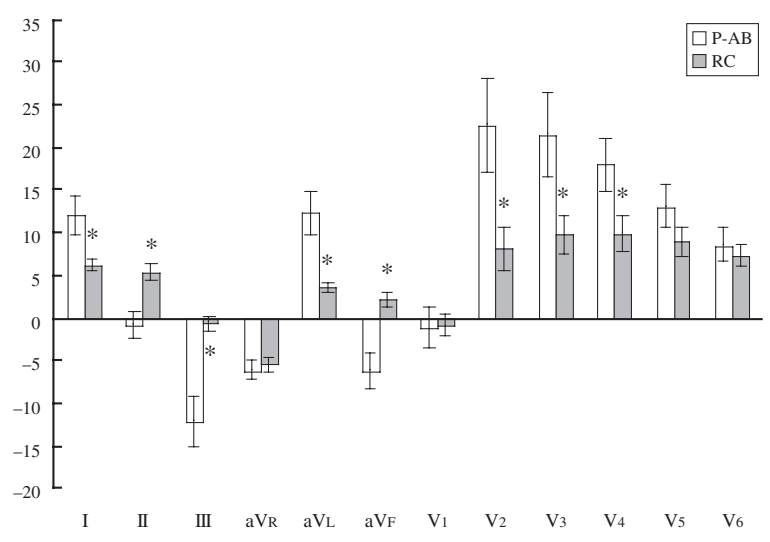

Fig 3. Expression of long-term cardiac memory. Compared with the post-ablation ECG (P-AB), the mean value of the T-wave areas of the recovery ECGs (RC) in leads II and aVF changed dramatically from negative to positive while that in lead III became significantly less negative. In addition, the mean value of the T-wave areas of $\mathrm{RC}$ in leads $\mathrm{I}, \mathrm{aV}, \mathrm{V}_{2-4}$ became significantly less positive when compared with those of $\mathrm{P}-\mathrm{AB} .{ }^{*} \mathrm{p}<0.05$ compared with $\mathrm{P}-\mathrm{AB}$. Error bars are mean \pm SEM.

\section{Results}

All patients had successful catheter ablation and AP conduction was completely abolished without complications. Five patients had a right posterior AP, 3 had a right posterior septal AP, 1 had a right lateral AP and 2 had a left posterior AP. The mean heart rate after catheter ablation was not significantly different from that immediately after RVOT pacing ( $72 \pm 6$ vs $74 \pm 8$ beats/min; $p>0.05$ ). Tables 1 and 2 show the location of the AP and changes in QRS polarity in each lead after AP ablation.

\section{Expression of Long-Term CM}

Tables 3 and 4 demonstrate the mean T-wave areas in the post-ablation and recovery ECGs, and the expression of long-term $\mathrm{CM}$ in each lead in all 11 patients. The T-wave areas of the post-ablation ECGs were significantly different from those of the recovery ECGs in each lead in all patients because of partial recovery from long-term CM (Figs 2B,E). Immediately after catheter ablation, the $\mathrm{T}$-wave areas were dominantly negative in leads III, $\mathrm{a} \mathrm{V}_{\mathrm{R}}, \mathrm{aVF}$, and dominantly positive in leads I, $\mathrm{aV}_{\mathrm{L}}$ and $\mathrm{V}_{2-6}$, whereas those in leads II and $\mathrm{V}_{1}$ were in between. In comparison with the post-ablation ECGs, the mean value of the T-wave areas of the recovery ECGs in leads II and aVF changed dramatically from negative to positive, reflecting a statistically significant increase $(\mathrm{p}<0.01)$, whereas that in lead III became significantly less negative $(p<0.01)$ (Fig 3$)$. In addition, the mean value of the T-wave areas of the recovery ECGs in leads $\mathrm{I}, \mathrm{aV}_{\mathrm{L}}, \mathrm{V}_{2}-4$ became significantly less positive when compared with the post-ablation ECGs $(\mathrm{p}<0.05)$ (Fig 3). The T-wave changes between the post-ablation and recovery ECGs indicated that long-term CM was expressed after catheter ablation and that the significant changes in the Twaves of each ECG lead at 1 week of catheter ablation was because of the gradual loss of long-term CM. As demonstrated in Tables 3 and 4 , the quantitative expression of long-term $\mathrm{CM}$ can be calculated as the $\mathrm{T}$-wave areas of $\{(\mathrm{P}-\mathrm{AB})-(\mathrm{RC})\}$.

Effect of Short-Term CM on Expression of Long-Term CM

As demonstrated in Tables 5 and 6, the T-wave areas of the post-pacing ECGs in each lead were significantly different from those of the post-ablation ECGs in all patients because of the effect of short-term CM induced by pacing from RVOT (Figs 2C,D). Compared with the post-ablation ECGs, the mean value of the T-wave areas of the postpacing ECGs in lead II changed dramatically from negative to positive, reflecting a statistically significant increase, whereas those in leads III and $\mathrm{aVF}$ became significantly less negative $(\mathrm{p}<0.01)(\mathrm{Fig} 4)$. The mean value of the $\mathrm{T}$-wave area of the post-pacing ECGs in leads I, a $\mathrm{V}_{\mathrm{L}}, \mathrm{V}_{2}-4$ were all significantly less positive than those of the post-ablation ECGs $(\mathrm{p}<0.05)($ Fig 4). As demonstrated in Tables 5 and 6 , the quantitative expression of the effect of short-term $\mathrm{CM}$ 
Table 5 T-Wave Areas of the Post-Ablation and Post-Pacing ECGs, and the ESCM in the Limb Leads

\begin{tabular}{|c|c|c|c|c|c|c|c|c|c|c|c|c|c|c|c|c|c|c|}
\hline \multirow{2}{*}{ Case no. } & \multicolumn{3}{|c|}{$I\left(\mathrm{~mm}^{2}\right)$} & \multicolumn{3}{|c|}{$I I\left(m^{2}\right)$} & \multicolumn{3}{|c|}{ III $\left(\mathrm{mm}^{2}\right)$} & \multicolumn{3}{|c|}{$a V_{R}\left(m^{2}\right)$} & \multicolumn{3}{|c|}{$a V_{L}\left(m^{2}\right)$} & \multicolumn{3}{|c|}{$a V_{F}\left(m m^{2}\right)$} \\
\hline & $P-A B$ & $P-P A$ & ESCM & $-A B$ & $P-P A$ & ESCM & $P-A B$ & $P-P A$ & $E S C M$ & $P-A B$ & $P-P A$ & ESCM & $P-A B$ & $P-P A$ & $E S C M$ & $P-A B$ & $P-P A$ & $E S C M$ \\
\hline 1 & 31 & 9.52 & & 37 & 2.77 & 1. & 2.46 & -6.51 & 5.59 & .97 & -6.26 & 1. & 4.63 & 8.38 & 25 & -5.51 & -1.62 & 3.89 \\
\hline 2 & 11.17 & 5.62 & J & 35 & 1.55 & 1. & .75 & -5.58 & 4.17 & -7.57 & -3.60 & 3.97 & 10.07 & 5.23 & 84 & -4.63 & -2.46 & 2.17 \\
\hline 3 & 31.61 & 24.01 & -7.60 & -10.8 & -8.99 & 1.81 & -39.78 & -30.37 & 9.41 & -11.18 & -7.47 & 3.71 & 37.18 & 29.04 & -8.14 & -23.52 & -18.57 & 4.95 \\
\hline 4 & 16.86 & 10.75 & -6.11 & 7.01 & 7.50 & 0.49 & -8.3 & -3.54 & 4.76 & -12.78 & -8.57 & 4.21 & 13.19 & 6.87 & -6.32 & -1.48 & 1.40 & 2.88 \\
\hline 5 & 7.21 & 6.15 & -1.06 & 4.48 & 5.03 & 0.55 & -2.98 & -1.52 & 1.46 & -5.56 & -5.76 & -0.20 & 5.20 & 3.75 & & 2.23 & 2.25 & 0.02 \\
\hline 6 & 56 & 8.14 & -0.52 & 3.04 & 0.0 & 3.04 & -11.4 & -7.26 & 4.14 & -3.38 & -3.94 & -0.56 & 9.91 & 8.16 & & -7.65 & -3.90 & 3.75 \\
\hline 7 & & 5.36 & 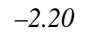 & & 6.0 & & 85 & -3.09 & 1. & -6.62 & -4.69 & & 5.66 & & & 0.40 & 2.56 & 2.16 \\
\hline 8 & 11.80 & 9.0 & -2.80 & 2.35 & -1.46 & 0.89 & -13.47 & -8.89 & 4.58 & -5.42 & -4.0 & 1.42 & 12.83 & 8.93 & -3.90 & -7.58 & -5.08 & 2.50 \\
\hline 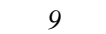 & .02 & 3.88 & -2.14 & -3.27 & 0.59 & 3.86 & -7.43 & -3.32 & 4.11 & -1.68 & -2.97 & -1.29 & 7.79 & 3.73 & -4.06 & -5.34 & -2.12 & 3.22 \\
\hline 10 & 6.62 & 5.82 & -0.80 & -6.33 & -4.27 & 2.06 & -12.55 & -9.05 & 3.50 & 0.0 & -0.74 & -0.74 & 8.99 & 7.05 & -1.94 & -9.89 & -6.43 & 3.46 \\
\hline 11 & 9.19 & 7.16 & -2.03 & -1.60 & 4.90 & 6.50 & -9.63 & -3.19 & 6.44 & -5.36 & -6.17 & -0.81 & 10.16 & 4.89 & -5.27 & -5.29 & 1.27 & 6.56 \\
\hline Mean & 12.09 & 8.67 & -3.42 & -0.77 & 1.24 & 2.01 & -12.05 & -7.48 & 4.57 & -6.14 & -4.92 & 1.21 & 12.33 & 8.23 & -4.09 & -6.21 & -2.97 & 2.23 \\
\hline SEM & 2.24 & 1.65 & 0.78 & 1.60 & 1.47 & 0.56 & 2.94 & 2.41 & 0.66 & 1.14 & 0.69 & 0.62 & 2.64 & 2.16 & 0.70 & 2.04 & 1.80 & 0.54 \\
\hline$p$ value & 0.003 & & & 0.003 & & & 0.003 & & & 0.11 & & & 0.003 & & & 0.003 & & \\
\hline
\end{tabular}

P-PA, post-right ventricular outlet tract pacing; ESCM, effects of short-term cardiac-memory on LCM. Other abbreviations as in Tables 1,3.

$E S C M=(P-P A)-(P-A B) . P$ value of comparison between $P-A B$ and $P-P A$.

Table 6 T-Wave Areas of the Post-Ablation and Post-Pacing ECGs, and the ESCM in the Precordial Leads

\begin{tabular}{|c|c|c|c|c|c|c|c|c|c|c|c|c|c|c|c|c|c|c|}
\hline \multirow{2}{*}{ Case no. } & \multicolumn{3}{|c|}{$\overline{V_{l}\left(m m^{2}\right)}$} & \multicolumn{3}{|c|}{$V_{2}\left(\mathrm{~mm}^{2}\right)$} & \multicolumn{3}{|c|}{$V_{3}\left(\mathrm{~mm}^{2}\right)$} & \multicolumn{3}{|c|}{$V_{4}\left(m^{2}\right)$} & \multicolumn{3}{|c|}{$V_{5}\left(\mathrm{~mm}^{2}\right)$} & \multicolumn{3}{|c|}{$V_{6}\left(\mathrm{~mm}^{2}\right)$} \\
\hline & $P-A B$ & $P-P A$ & $\overline{E S C M}$ & $A B$ & $P-P A$ & $\overline{E S C M}$ & $A B$ & $P-P A$ & $E S C M$ & $A B$ & $P-P A$ & $E S C M$ & $-A B$ & $P-P A$ & $\overline{E S C M}$ & $-A B$ & $P-P A$ & $E S C M$ \\
\hline 1 & 57 & -5.79 & 122 & 61 & 19.83 & 878 & 7.17 & 81 & 36 & 63 & 72 & 21 & 70 & 77 & 93 & 7.91 & 0.79 & 7.12 \\
\hline 2 & -5.04 & 4.33 & & 15 & 4.92 & -9 & & 4.81 & 17.30 & 21.11 & 9.29 & -11.82 & .13 & 8.90 & 23 & 50 & 7.03 & -7.47 \\
\hline 3 & & 3.1. & -0.19 & 55 & 38.01 & -9 & & 39 & 28 & 2. & 19.46 & 71 & 58 & 29 & & & 13.98 & -3.75 \\
\hline 4 & - & -4.36 & 1.06 & 6.89 & 27.66 & -9.23 & & 27.62 & -12.96 & 33.27 & 23.34 & -9.93 & 0.84 & 60 & & 05 & & -4.47 \\
\hline 5 & -3.72 & -3.79 & -0.07 & 19.55 & 16.80 & -2.75 & 10.82 & 10.15 & -0.67 & 9.05 & 9.13 & 0.08 & 7.00 & 6.27 & -0.73 & 6.87 & 5.03 & -1.84 \\
\hline 6 & 16.48 & 4.13 & -12.35 & 46.91 & 20.54 & -26.37 & 31.83 & 13.01 & -18.82 & 17.63 & 10.09 & -7.54 & 7.29 & 7.20 & -0.09 & 2.65 & 4.22 & 1.57 \\
\hline 7 & -8.48 & -7.35 & 1.13 & -6.75 & -10.14 & -3.39 & 0.9 & -5.32 & -6.22 & 9.68 & 0.53 & -9.15 & 13.29 & 9.04 & -4.25 & 11.31 & 9.60 & -1.71 \\
\hline 8 & -1.10 & 2.13 & 3.23 & 31.41 & 27.14 & -4.27 & 30.94 & 24.24 & -6.70 & 22.56 & 18.27 & -4.29 & 12.27 & 9.59 & -2.68 & 6.10 & 5.22 & -0.88 \\
\hline 9 & & 5.99 & -2.93 & & 15.02 & -5.82 & & 11.70 & -0.03 & 6.80 & 9.84 & 3.04 & 4.49 & 6.69 & 2.20 & 2.47 & 4.24 & 1.77 \\
\hline 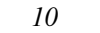 & 3.86 & 0.6 & -3.86 & 73 & 12.09 & -6 . & & 12. & -2.63 & 11.91 & 10.43 & -1.48 & 3.54 & 5.56 & 2.02 & -2.73 & & 4.48 \\
\hline 1 & 11.29 & -11.95 & -0.66 & 8.37 & -15.56 & -7.19 & -6.27 & -3.67 & 2.62 & 6.47 & 8.14 & 1.67 & 5.29 & 7.97 & 2.68 & 4.53 & 6.27 & 1.74 \\
\hline Mean & -1.21 & -2.59 & -1.38 & 22.68 & 14.21 & -8.47 & 21.63 & 13.69 & -7.94 & 18.03 & 13.36 & -4.67 & 13.13 & 10.26 & -2.87 & 8.58 & 6.97 & -1.61 \\
\hline SEM & 2.43 & 1.58 & 1.24 & 5.60 & 4.83 & 1.93 & 4.95 & 3.65 & 2.19 & 3.14 & 2.42 & 1.52 & 2.57 & 1.45 & 1.32 & 2.04 & 1.06 & 1.16 \\
\hline p value & 0.42 & & & 0.00 & & & 0.008 & & & 0.03 & & & 0.07 & & & 0.25 & & \\
\hline
\end{tabular}

Abbreviations as in Tables 1,5.

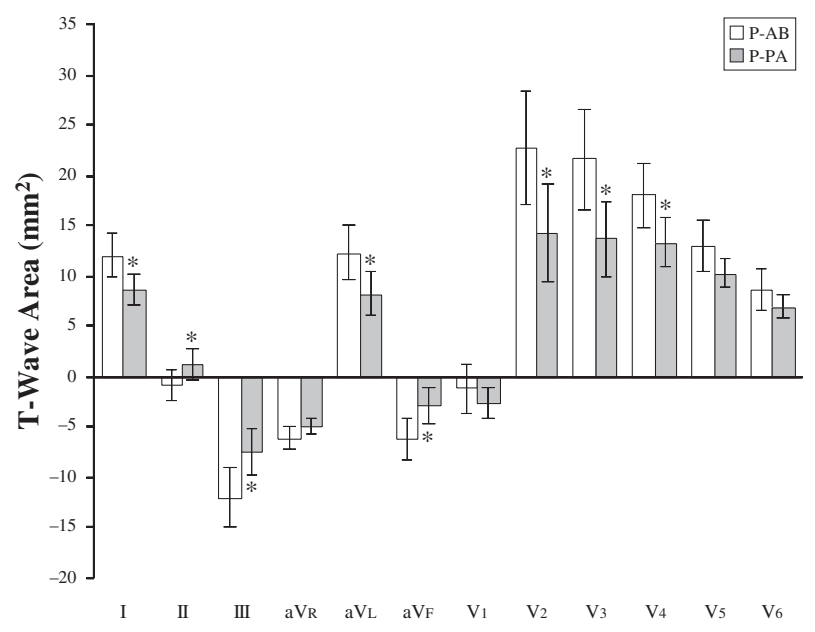

Fig 4. Effects of short-term cardiac memory $(\mathrm{CM})$ on expression of long-term CM. Compared with the T-wave areas in the post-ablation ECGs (P-AB), the T-wave areas in the post-pacing ECGs (P-PA) change significantly from negative to positive in lead II and become less negative in leads III and aVF. In addition, the T-wave areas become less positive in leads I, aVL, $\mathrm{V}_{2-4}$ in the post-pacing ECGs. $* \mathrm{p}<0.05$ compared with P-AB. Error bars are mean \pm SEM.

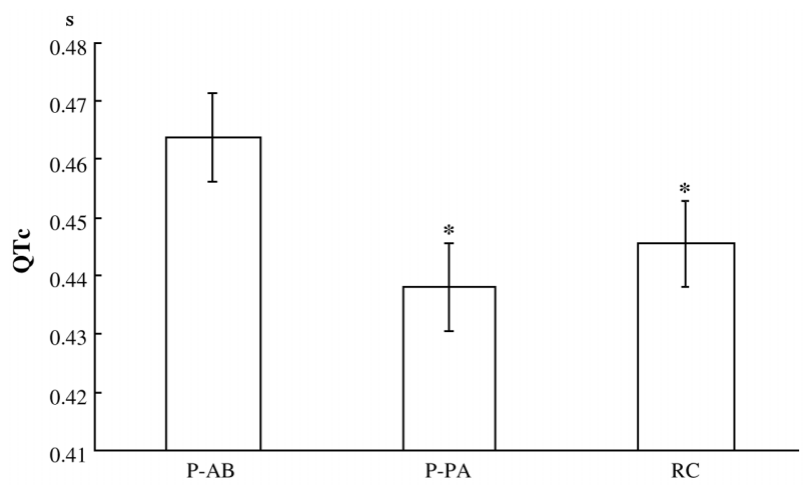

Fig 5. Mean QTc interval in the post-ablation, post-pacing and recovery ECGs. The mean QTc interval is significantly shorter in the post-pacing and recovery ECGs than in post-ablation ECGs. However, the mean QTc interval is not significantly different in the post-pacing and recovery ECGs. P-AB, post-ablation ECG; P-PA, post-pacing ECG; RC, recovery ECG. *p $<0.05$ as compared with post-ablation EC. 
on long-term $\mathrm{CM}$ can be calculated as the T-wave areas of $\{(\mathrm{P}-\mathrm{PA})-(\mathrm{P}-\mathrm{AB})\}$. Of note, all the effects of short-term $\mathrm{CM}$ induced by $20 \mathrm{~min}$ of RVOT pacing on the expression of long-term CM were absent and the post-ablation ECG pattern resumed within $30 \mathrm{~min}$.

\section{Changes of QTC in Short- and Long-Term CM}

The mean maximal QTc in the post-ablation, post-pacing and recovery ECGs was $0.47 \pm 0.01,0.44 \pm 0.01$ and $0.45 \pm$ $0.01 \mathrm{~s}$, respectively. The QTc in post-ablation ECG was significantly longer than in either the post-pacing or recovery ECGs ( $\mathrm{p}=0.03$ and 0.02 respectively). The QTc in the postpacing ECG was not significantly different from that in the recovery ECG (p=0.37) (Fig 5).

\section{Discussion}

\section{Major Findings}

In patients with WPW syndrome, long-term CM expressed as changes in the T-wave area and prolongation of the QTc can be demonstrated after catheter ablation of AP conduction. Short-term CM induced by pacing from the RVOT can counteract the expression of long-term CM by decreasing the degree of the negative $\mathrm{T}$-wave area in leads II, III, $\mathrm{aVF}$ and shortening of the QTc.

\section{Interaction of Long- and Short-Term CM}

Although CM has been well studied in animal models, the mechanisms of short- and long-term CM in humans is still uncertain. In a canine study, the protein inhibitor cycloheximide markedly attenuated the evolution of CM induced by 3 weeks of ventricular pacing and this was referred to as long-term CM${ }^{16}$ Four-aminopyridine can alter the T-wave changes that persist after a short period of ventricular pacing, and this is referred to as short-term CM in the canine heart! ${ }^{13}$ The ICaL blocker can suppress the CM induced by both short- and long-term ventricular pacing! ${ }^{17}$ Angiotensin-converting enzyme inhibitors and angiotensinII receptor blockers reportedly suppressed short-term rather than long-term CM in a canine study ${ }^{17,18}$ Recently, Janse et al reported that the apicobasal gradients in repolarization are important in the genesis of the T-wave and that CM may induce a more rapid phase 3 of the action potential 15 The results from these animal studies suggest that the mechanism of $\mathrm{CM}$ is a complex process involving ion-channel changes, gap junction changes and signal transduction changes in mRNA and protein synthesis, and that the mechanisms of short- and long-term CM might differ ${ }^{19}$ In humans, the changes in the T-wave in patients with manifest WPW syndrome after catheter ablation of AP conduction are typical presentations of long-term CM? These changes can persist for a few weeks after catheter ablation and in each lead are associated with the location of the AP? In the present study, we selected patients with WPW syndrome whose APs were located close to the posterior and septal wall so that markedly negative T-waves in leads II, III, and aVF after catheter ablation of AP conduction would be obtained.

Previous studies had demonstrated that the polarity of the $\mathrm{T}$-wave changes present in short-term CM correlate with the polarity of the QRS complex induced by RV pacing? In the present study, in order to induce short-term CM, we selected the RV pacing site at the RVOT, which would produce a positive polarity of the QRS complex during ventricular pacing and induce positive $\mathrm{T}$-wave changes (ie, short-term CM) in leads II, III and aVF. Of note, the results from our study clearly demonstrate that the positive T-wave changes induced by short-term CM can temporarily counteract the expression of long-term CM by decreasing the degree of negative T-waves in leads II, III and aVF. Another interesting finding is that the positive $\mathrm{T}$-waves present in leads $\mathrm{I}, \mathrm{aV}$ and $\mathrm{V}_{2-4}$ after catheter ablation were significantly attenuated after 20 min of RVOT pacing. The reason for this attenuation in the $\mathrm{T}$-wave areas was the negative QRS complex in leads $\mathrm{I}, \mathrm{aV} \mathrm{L}$ and $\mathrm{V}_{2-4}$ during RVOT pacing, which produced short-term $\mathrm{CM}$ with negative $\mathrm{T}$ wave effect and decreased the amplitude and area of the previous positive T-waves. As mentioned earlier, mechanisms involving the expression of short- and long-term CM are complex and it is even more difficult to evaluate the exact mechanisms involved in human CM. However, the present results demonstrate that there is an interaction between short- and long-term CM and further studies are needed to evaluate the mechanisms.

\section{Changes of QTc in CM}

In an animal study, Plotnikov et al demonstrated that the QT interval was prolonged by a long period of 21 days' pacing; however, only minimal prolongation of the QT interval was induced by short period of $20 \mathrm{~min}$ of pacing from the LV20 In the present study, immediately after catheter ablation, the QTc was significantly longer than after either RVOT pacing or in the recovery ECGs. This prolongation of the QTc can be explained by the effects of long-term CM induced by ventricular preexcitation. The long-lasting abnormal ventricular activation sequence caused by preexcitation may induce a ventricular gradient and the dispersion of ventricular repolarization may be increased immediately after catheter ablation, which results in an altered ventricular activation sequence. ${ }^{1}$ In the recovery ECG, the QTc was significantly shortened because of gradual loss of the effect of long-term CM. In addition, after pacing from the RVOT for $20 \mathrm{~min}$, the QTc of long-term CM was also significantly shortened, which suggests that short-term CM not only can induce shortening of the QTc but also can counteract the prolongation of the QTc caused by long-term CM. Whether the dynamic changes in the QTc interval during the evolution of CM are the genesis of ventricular arrhythmogenicity needs further study.

\section{Clinical Implications}

Nonspecific ST-T wave and dynamic QT interval changes are common and complicated ECG presentations. In addition to the conventional factors that have been reported to cause these changes, the effects of long- and short-term CM and their interaction are possible new explanations. Whether the CM expressed as dynamic QT interval changes is a possible explanation of the ventricular arrhythmia and sudden cardiac death that occur in patients with WPW syndrome needs further investigation.

\section{Study Limitations}

First, the number of study patients was limited. However, the effect of short-term CM on long-term CM was consistent in leads II, III, and aVF in all patients. Second, longterm CM usually persisted for a few weeks after catheter ablation. The ECG obtained at 1 week after catheter ablation was not the true "baseline" ECG. Therefore, we could not demonstrate the true expression of long-term CM. Third, short-term CM was induced by pacing from RVOT 
in all patients but one, in whom short-term $\mathrm{CM}$ was induced by pacing from the RVA. Whether the pacing from other sites in the RV would result in the same effects on longterm $\mathrm{CM}$ requires further evaluation.

\section{Conclusions}

Long-term CM can be demonstrated in patients with manifest WPW syndrome immediately after catheter ablation of AP conduction. Short-term CM induced by a short period of pacing from the RVOT is able to interfere with the expression of long-term CM, resulting in dramatic T-wave changes and QTc changes. These results indicate that the expression of long-term CM is affected by shortterm CM.

\section{References}

1. Nicolai P, Medvedowsky JL, Delaage M, Barnay C, Blache E, Pisapia A. Wolff-Parkinson-White syndrome: T wave abnormalities during normal pathway conduction. J Electrocardiol 1981; 14: 295 300.

2. Kalbfleisch SJ, Sousa J, EI-Atassi R, Calkins H, Langberg J, Morady F. Repolarization abnormalities after catheter ablation of accessory atrioventricular connections with radiofrequency current. J Am Coll Cardiol 1991; 18: 1761-1766.

3. Wood MA, DiMarco JP, Jaines DE. Electrocardiographic abnormalities after radiofrequency catheter ablation of accessory bypass tracts in the Wolff-Parkinson-White syndrome. Eur Heart J 1992; 13: $801-807$.

4. Chatterjee K, Harris AM, Davies JG, Leatham A. T-wave changes after artificial pacing. Lancet 1969; 1: 759-760.

5. Rosenbaum MB, Blanco HH, Elizari MV, Lazzarri JO, Davidenko $\mathrm{JM}$. Electrotonic modulation of the T wave and cardiac memory. Am J Cardiol 1982; 50: 213-222.

6. Goyal R, Syed ZA, Mukhopadhyay PS, Souza J, Zivin A, Knight BP, et al. Changes in cardiac repolarization following short periods of ventricular pacing. J Cardiovasc Electrophysiol 1998; 9: 269-280.

7. Costard-Jackle A, Goetsch B, Antz M, Franz MR. Slow and longlasting modulation of myocardial repolarization produced by ectopic activation in isolated rabbit hearts. Circulation 1998; 80: $1412-$
1420

8. Rosenbaum MB, Balnco HH, Elizari MV. Electrocardiographic characteristics and main causes of pseudoprimary $\mathrm{T}$ wave changes: Significance of concordant and discordant $\mathrm{T}$ wave in the human and other animal species. Ann NY Acad Sci 1990; 601: 36-50.

9. Chatterjee K, Harris A, Davies G, Leatham A. Electrocardiographic changes subsequent to artificial ventricular depolarization. Br Heart $J$ 1969; 31: 770-779.

10. Gould L, Venkataraman K, Goswami MK, Gomprecht RF. Pacemaker induced electrocardiographic changes simulating myocardial infarction. Chest 1973; 63: 829-833.

11. Lab MJ. Contraction-excitation feedback in myocardium: Physiological basis and clinical relevance. Circ Res 1982; 50: 757-766.

12. Katz AM. T wave "memory": Possible causal relationship to stressinduced changes in cardiac ion channels? J Cardiovasc Electrophysiol 1992; 3: 150-159.

13. del Balzo U, Rosen MR. T wave changes persisting after ventricular pacing in canine heart are altered by 4-aminopyridine but not by lidocaine. Circulation 1992; 85: 1464-1472.

14. Patberg KW, Rosen MR. Molecular determinants of cardiac memory and their regulation. J Mol Cell Cardiol 2004; 36: 195-204.

15. Janse MJ, Sosunov EA, Coronel R, Opthof T, Anyukhovsky EP, de Bakker JM, et al. Repolarization gradients in the canine left ventricle before and after induction of short-term cardiac memory. Circulation 2005; 112: $1711-1718$.

16. Shvilkin A, Danilo P Jr, Wang J, Burkhoff D, Anyukhovsky EP, Sosunov EA, et al. Evolution and resolution of long-term cardiac memory. Circulation 1998; 97: 1810-1817.

17. Plotnikov AN, Yu H, Geller JC, Gainullin RZ, Chandra P, Patberg $\mathrm{KW}$, et al. Role of L-type calcium channels in pacing-induced shortterm and long-term cardiac memory in canine heart. Circulation 2003; 107: 2844-2849.

18. Ricard P, Danilo P Jr, Cohen IS, Burkhoff D, Rosen MR. A role for the rennin-angiotensin system in the evolution of cardiac memory. $J$ Cardiovasc Electrophysiol 1999; 10: 545-551.

19. Patel PM, Plotnikov A, Kanagratnam P, Shvilkin A, Sheehan CT, Xiong W, et al. Altering ventricular activation remodels gap junction distribution in canine heart. J Cardiovasc Electrophysiol 2001; 12: 570-577.

20. Plotnikov AN, Shvilkin A, Xiong W, deGroot JR, Rosenshtraukh L, Feinmark S, et al. Interactions between antiarrhythmic drugs and cardiac memory. Cardiovasc Res 2001; 50: 335-344.

21. Kurita T, Ohe T, Maeda K, Isobe F, Kamakura S, Shimomura K. QRS alteration-induced torsade de pointes in a patient with an artifical pacemaker and hypokalemia. Jpn Circ J 1996; 60: 189-191. 